\title{
Regioselective ring opening of 2,2-dicyanooxiranes by 1,3-dinucleophiles in the presence of Lewis acids such as bismuth(III) nitrate pentahydrate $\left[\mathrm{Bi}\left(\mathrm{NO}_{3}\right)_{3} \cdot 5 \mathrm{H}_{2} \mathrm{O}\right]$ and zirconium(IV) chloride $\left(\mathrm{ZrCl}_{4}\right)$
}

\author{
Mahboobeh Karbalaei • Mohammad Seifi • \\ Hassan Sheibani
}

Received: 29 December 2013/Accepted: 8 February 2014/Published online: 6 March 2014

(C) The Author(s) 2014. This article is published with open access at Springerlink.com

\begin{abstract}
Bismuth(III) nitrate pentahydrate $\left[\mathrm{Bi}\left(\mathrm{NO}_{3}\right)_{3} \cdot 5 \mathrm{H}_{2} \mathrm{O}\right]$ or zirconium(IV) chloride $\left(\mathrm{ZrCl}_{4}\right)$ has been shown to catalyze nucleophilic ring opening of 2,2dicyanooxiranes along with ring closure by 1,3-dinucleophiles such as $1 \mathrm{H}-1,3$ benzimidazole-2-thiol, 5-phenyl-4H-1,2,4-triazole-3-thiol, and thioureas. These reactions led to efficient synthesis of heterocyclic compounds condensed with benzimidazole or triazole derivatives. The used catalysts are inexpensive, highly efficient, and reusable for opening of epoxides with 1,3-dinucleophiles at ambient temperature, with excellent regioselectivity.
\end{abstract}

Keywords Bismuth(III) nitrate pentahydrate $\left[\mathrm{Bi}\left(\mathrm{NO}_{3}\right)_{3} \cdot 5 \mathrm{H}_{2} \mathrm{O}\right]$.

Zirconium(IV) chloride $\cdot 1 H$-1,3-Benzimidazole-2-thiol $\cdot$ 5-Phenyl-4H-1,2,4-

triazole-3-thiol $\cdot$ Thioureas $\cdot$ 2,2-Dicyanooxiranes

\section{Introduction}

2,2-Dicyanooxiranes and their $\alpha$-haloketone derivatives are known as very effective bielectrophile reagents for synthesis of a large variety of heterocyclic compounds such as thiazoles [1], dithioles [2], imidazoles [3], 1,3-oxathioles [4], and condensed imidazolo and thiazolo derivatives [5]. In general, nucleophilic reagents can react with these compounds regioselectively: they can attack the oxirane ring, cyano functional group to give either ring-opened products, new functionalized oxiranes or different heterocycles. Epoxide ring opening with nucleophilic agents is an important step in preparation of 1,2-disubstituted products [6]. Heating epoxides in the presence of nucleophiles, which would occur with poorly nucleophilic addition, suffers from lack of appreciable regioselectivity [6]. The requirement for high

M. Karbalaei · M. Seifi $\cdot$ H. Sheibani $(\bowtie)$

Department of Chemistry, Shahid Bahonar University of Kerman, 76169 Kerman, Iran e-mail: hsheibani@mail.uk.ac.ir 
temperature in epoxides sensitive to this condition led to the necessity for activation of the epoxide rings to increase their susceptibility to nucleophilic attack. The various methodologies developed for this purpose include the use of Lewis acid catalysts such as alumina [7], metal triflates [8], transition-metal halides [9], alkalimetal perchlorates [10], silica under high pressure [11], and montmorillonite clay under microwave irradiation [12]. The search for new and efficient Lewis acid catalysts for various useful organic transformations is one of the most important, interesting, and challenging research topics in catalytic synthesis. On the other hand, in recent years, economically affordable ecofriendly catalysts have received greater acceptance for use in organic reactions. In this respect, bismuth(III) and zirconium(IV) derivatives are known as Lewis acid catalysts in various organic transformations involving carbon-carbon bond formation, protection-deprotection chemistry, and conversion of epoxides into vic-acylamino hydroxy compounds [1316]. Since zirconium, in its normal quadrivalent state, displays no redox properties but can attain a maximum covalency up to eight and displays low toxicity [16], $\mathrm{Zr}(\mathrm{IV})$ compounds should be ideal for catalytic applications. Coordination of $\mathrm{Bi}^{3+}$ with the epoxide oxygen renders the epoxide susceptible to nucleophilic attack by nucleophilic reagents. Considering the widespread applications of 2,2-dicyanooxiranes in synthesis of a large variety of heterocyclic compounds and their sensitivity to high temperature, we planned to employ bismuth(III) nitrate pentahydrate and zirconium(IV) chloride as Lewis acid catalysts to evaluate their catalytic efficiency in carrying out ring-opening reactions of these epoxides with 1,3-dinucleophiles.

\section{Results and discussion}

We investigated ring opening and condensation reaction of 2,2-dicyanooxiranes (1a-1d) with 1,3-dinucleophiles such as 1H-1,3-benzimidazole-2-thiol (2), 5-phenyl-4H-1,2,4-triazole-3-thiol (4), and thioureas $(\mathbf{6 a}, \mathbf{6 b})$ in different conditions at ambient temperature (Scheme 1). Lewis acid catalysts, namely bismuth(III) nitrate pentahydrate and zirconium(IV) chloride, were used for activation of the epoxide rings, rendering them more susceptible to nucleophilic attack under milder conditions. Selective formation of the regioselective products $\mathbf{3}, \mathbf{5}$, and $\mathbf{7}$ arising from nucleophilic attack at the benzylic carbon $\left(\mathrm{C}_{\beta}\right)$ of 2,2-dicyanooxiranes (1a-1d) was observed in the course of the reactions with 1,3-dinucleophiles $\mathbf{2}, \mathbf{4}$, and $\mathbf{6}$.

Reactions of 2,2-dicyanooxiranes with 1,3-dinucleophiles occur at ambient temperature in the absence of catalyst in long experimental times [17-19]. These epoxides are sensitive to high temperature; therefore, we planned to employ Lewis acid catalysts bismuth(III) nitrate pentahydrate and zirconium(IV) chloride to decrease the activation energy of the ring-opening reaction of epoxides with 1,3dinucleophiles (Table 1).

To determine standard operating conditions for these reactions, we used some polar and nonpolar solvents in the reaction of 3-phenyloxirane-2,2-dicarbonitrile (1a) and $1 H$-benzo $[d]$ imidazole-2-thiol (2) in the presence of Lewis acid catalysts bismuth(III) nitrate pentahydrate and zirconium(IV) chloride as model reactions to 
<smiles>[R4]c1cc([R1])c(C2OC2(C)C#N)cc1[R4]</smiles>

1a-d<smiles>[R]c1cc([R])c(C2OC2(C#N)C#N)cc1[R]</smiles>

$1 b$ and 1d<smiles>COc1ccc(OC)c(C2OC2(C#N)C#N)c1</smiles>

1d<smiles>Sc1nc2ccccc2[nH]1</smiles>

2<smiles>Sc1nnc(-c2ccccc2)[nH]1</smiles>

4<smiles>[R]NC(N)=S</smiles>

6a, b<smiles>[R]c1cc([R])c(C2Sc3nc4ccccc4n3C2=O)cc1[R]</smiles>

3a-d

1a, 3a: $\mathrm{R}_{1}=\mathrm{R}_{2}=\mathrm{R}_{3}=\mathrm{H}$

1c, 3c: $\mathrm{R}_{1}=\mathrm{R}_{3}=\mathrm{H}, \mathrm{R}_{2}=\mathrm{NO}_{2}$

1b, 3b: $\mathrm{R}_{1}=\mathrm{R}_{3}=\mathrm{H}, \mathrm{R}_{2}=\mathrm{Cl}$

1d, 3d: $\mathrm{R}_{1}=\mathrm{R}_{3}=\mathrm{OCH}_{3}, \mathrm{R}_{2}=\mathrm{H}$

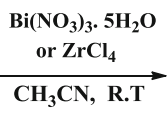

$$
\begin{gathered}
\underset{\mathrm{Bi}\left(\mathrm{NO}_{3}\right)_{3} \cdot 5 \mathrm{H}_{2} \mathrm{O}}{\text { or } \mathrm{ZrCl}_{4}} \\
\underset{\mathrm{CH}_{3} \mathrm{CN}, \mathrm{R} . \mathrm{T}}{\longrightarrow}
\end{gathered}
$$<smiles></smiles>

$7 \mathrm{a}, \mathrm{b}$

6a, 7a: $\mathrm{R}=\mathrm{H}$

6b, 7b: $\mathrm{R}=\mathrm{Ph}$

Scheme 1 Condensation reaction of 2,2-dicyanooxiranes (1a-1d) with 1,3-dinucleophiles such as $1 H$ 1,3-benzimidazole-2-thiol (2), 5-phenyl-4H-1,2,4-triazole-3-thiol (4), and thioureas (6a, 6b)

Table 1 Reaction of 1,3-dinucleophiles $(\mathbf{2}, \mathbf{4}, \mathbf{6 a}, \mathbf{6 b})$ with 2,2-dicyanooxiranes (1a-1d) for synthesis of

\begin{tabular}{|c|c|c|c|c|c|c|c|c|}
\hline \multirow[t]{2}{*}{ Compd. } & \multirow[t]{2}{*}{ Oxirane } & \multirow[t]{2}{*}{ Dinuc. } & \multicolumn{2}{|c|}{ Without catalyst } & \multicolumn{2}{|c|}{$\mathrm{Bi}\left(\mathrm{NO}_{3}\right)_{3}(5 \mathrm{~mol} . \%)$} & \multicolumn{2}{|c|}{$\mathrm{ZrCl}_{4}(10 \mathrm{~mol} . \%)$} \\
\hline & & & Time (h) & Yield (\%) & Time (min) & Yield (\%) & Time (min) & Yield (\%) \\
\hline $\mathbf{3 a}$ & $1 \mathbf{a}$ & 2 & 7 & 90 & 40 & 90 & 30 & 92 \\
\hline $\mathbf{3 b}$ & $\mathbf{1 b}$ & 2 & 7 & 93 & 35 & 92 & 25 & 94 \\
\hline $3 c$ & 1c & 2 & 7 & 94 & 35 & 95 & 25 & 95 \\
\hline 3d & 1d & 2 & 7 & 90 & 40 & 93 & 35 & 94 \\
\hline $5 \mathbf{a}$ & $1 b$ & 4 & 7 & 90 & 30 & 92 & 25 & 92 \\
\hline $5 \mathbf{b}$ & 1d & 4 & 7 & 94 & 35 & 94 & 30 & 94 \\
\hline $7 \mathbf{a}$ & 1d & $6 \mathbf{a}$ & 24 & 68 & 70 & 83 & 60 & 85 \\
\hline $7 \mathbf{b}$ & 1d & $\mathbf{6 b}$ & 24 & 70 & 75 & 88 & 70 & 90 \\
\hline
\end{tabular}
3a-3d, 5a, 5b, 7a, and $7 \mathbf{b}$ in the presence of $\mathrm{Bi}\left(\mathrm{NO}_{3}\right)_{3} \cdot 5 \mathrm{H}_{2} \mathrm{O}(5 \mathrm{~mol} . \%)$ and $\mathrm{ZrCl}_{4}(10 \mathrm{~mol} . \%)$

investigate the effects of solvent for preparing compound 3a. In each case, the substrates were mixed together with $10 \mathrm{~mol} \%$ of these catalysts agitated with $10 \mathrm{~mL}$ solvent at room temperature. The results are presented in Table 2 . It is 
noteworthy that polar solvents such as acetonitrile afforded better yields than nonpolar solvents.

We also optimized the quantity of catalysts. The best results were obtained when the reactions were carried out in the presence of 5 or 10 mol.\% bismuth(III) nitrate pentahydrate and zirconium(IV) chloride, respectively. The results are presented in Table 3.

We also attempted to reuse the catalysts by a variety of methods (Table 4). Direct reuse of the catalysts (Table 4 , entry 2,6 ) led to a greater than $15 \%$ decrease in activity, while washing with dichloromethane and ethylacetate prior to reuse also resulted in lower conversions (Table 4, entry 3, 4, 7, 8). This phenomenon probably arose because the reactant and product were not completely desorbed from the catalyst, and therefore the active sites were blocked.

Using this protocol (Scheme 1), we investigated reactions of 2,2-dicyanooxiranes 1, which have three electron-deficient centers, with 1,3-dinucleophiles for synthesis of heterocyclic compounds in the presence of Lewis acid catalysts $\mathrm{Bi}\left(\mathrm{NO}_{3}\right)_{3} \cdot 5 \mathrm{H}_{2} \mathrm{O}$ (5 mol.\%) and $\mathrm{ZrCl}_{4}(10 \mathrm{~mol} \%$ ). In these reactions, the 1,3-dinucleophiles act

Table 2 Solvent effects on ring-opening reaction of epoxide 1a with $1 H$-benzo $[d]$ imidazole-2-thiol (2)

\begin{tabular}{|c|c|c|c|c|}
\hline Entry & Solvent & Catalyst & Time (min) & Yield $(\%)$ \\
\hline 1 & Toluene & $\mathrm{Bi}\left(\mathrm{NO}_{3}\right)_{3} \cdot 5 \mathrm{H}_{2} \mathrm{O}$ & 80 & 75 \\
\hline 2 & Toluene & $\mathrm{ZrCl}_{4}$ & 70 & 78 \\
\hline 3 & THF & $\mathrm{Bi}\left(\mathrm{NO}_{3}\right)_{3} \cdot 5 \mathrm{H}_{2} \mathrm{O}$ & 48 & 87 \\
\hline 4 & THF & $\mathrm{ZrCl}_{4}$ & 37 & 89 \\
\hline 5 & $\mathrm{CH}_{3} \mathrm{CN}$ & $\mathrm{Bi}\left(\mathrm{NO}_{3}\right)_{3} \cdot 5 \mathrm{H}_{2} \mathrm{O}$ & 40 & 90 \\
\hline 6 & $\mathrm{CH}_{3} \mathrm{CN}$ & $\mathrm{ZrCl}_{4}$ & 30 & 92 \\
\hline 8 & Ethanol & $\mathrm{Bi}\left(\mathrm{NO}_{3}\right)_{3} \cdot 5 \mathrm{H}_{2} \mathrm{O}$ & 45 & 88 \\
\hline 9 & Ethanol & $\mathrm{ZrCl}_{4}$ & 35 & 91 \\
\hline
\end{tabular}

THF tetrahydrofuran

Table 3 Optimized quantity of catalysts for ring-opening reaction of epoxide 1a in synthesis of compound 3a

\begin{tabular}{|c|c|c|c|c|}
\hline Entry & Catalyst & Mol.\% catalyst & Time (min) & Yield (\%) \\
\hline 1 & $\mathrm{Bi}\left(\mathrm{NO}_{3}\right)_{3} \cdot 5 \mathrm{H}_{2} \mathrm{O}$ & 3 & 50 & 85 \\
\hline 2 & $\mathrm{ZrCl}_{4}$ & 5 & 38 & 88 \\
\hline 3 & $\mathrm{Bi}\left(\mathrm{NO}_{3}\right)_{3} \cdot 5 \mathrm{H}_{2} \mathrm{O}$ & 4 & 45 & 87 \\
\hline 4 & $\mathrm{ZrCl}_{4}$ & 7.5 & 35 & 89 \\
\hline 5 & $\mathrm{Bi}\left(\mathrm{NO}_{3}\right)_{3} \cdot 5 \mathrm{H}_{2} \mathrm{O}$ & 5 & 40 & 90 \\
\hline 6 & $\mathrm{ZrCl}_{4}$ & 10 & 30 & 92 \\
\hline 7 & $\mathrm{Bi}\left(\mathrm{NO}_{3}\right)_{3} \cdot 5 \mathrm{H}_{2} \mathrm{O}$ & 6 & 40 & 90 \\
\hline 8 & $\mathrm{ZrCl}_{4}$ & 12.5 & 30 & 92 \\
\hline 9 & $\mathrm{Bi}\left(\mathrm{NO}_{3}\right)_{3} \cdot 5 \mathrm{H}_{2} \mathrm{O}$ & 7 & 40 & 90 \\
\hline 10 & $\mathrm{ZrCl}_{4}$ & 15 & 30 & 92 \\
\hline
\end{tabular}


Table 4 Reusability of $\mathrm{Bi}\left(\mathrm{NO}_{3}\right)_{3} \cdot 5 \mathrm{H}_{2} \mathrm{O}$ and $\mathrm{ZrCl}_{4}$ in ring-opening reaction of epoxide $\mathbf{1 a}$ for synthesis of compound 3a

\begin{tabular}{llll}
\hline Entry & Catalyst & Time (min) & Yield (\%) \\
\hline 1 & $\mathrm{Bi}\left(\mathrm{NO}_{3}\right)_{3} \cdot 5 \mathrm{H}_{2} \mathrm{O}$ (1st use) & 40 & 90 \\
2 & $\mathrm{Bi}\left(\mathrm{NO}_{3}\right)_{3} \cdot 5 \mathrm{H}_{2} \mathrm{O}^{\text {a }}$ (2nd use) & 70 & 75 \\
3 & $\mathrm{Bi}\left(\mathrm{NO}_{3}\right)_{3} \cdot 5 \mathrm{H}_{2} \mathrm{O}^{\mathrm{b}}$ (2nd use) & 55 & 83 \\
4 & ${\mathrm{Bi}\left(\mathrm{NO}_{3}\right)_{3} \cdot 5 \mathrm{H}_{2} \mathrm{O}^{\mathrm{c}} \text { (2nd use) }}_{2 \mathrm{ZrCl}_{4}(1 \text { st use) }}^{55}$ & 81 \\
5 & $\mathrm{ZrCl}_{4}^{\mathrm{a}}$ (2nd use) & 30 & 92 \\
6 & $\mathrm{ZrCl}_{4}^{\mathrm{b}}$ (2nd use) & 55 & 78 \\
7 & $\mathrm{ZrCl}_{4}^{\text {c }}$ (2nd use) & 40 & 85 \\
8 & 40 & 84 \\
\hline
\end{tabular}

${ }^{a}$ Direct reuse of catalysts

b $\mathrm{Bi}\left(\mathrm{NO}_{3}\right)_{3} \cdot 5 \mathrm{H}_{2} \mathrm{O}$ and $\mathrm{ZrCl}_{4}$ were washed with dichloromethane

c $\mathrm{Bi}\left(\mathrm{NO}_{3}\right)_{3} \cdot 5 \mathrm{H}_{2} \mathrm{O}$ and $\mathrm{ZrCl}_{4}$ were washed with ethylacetate

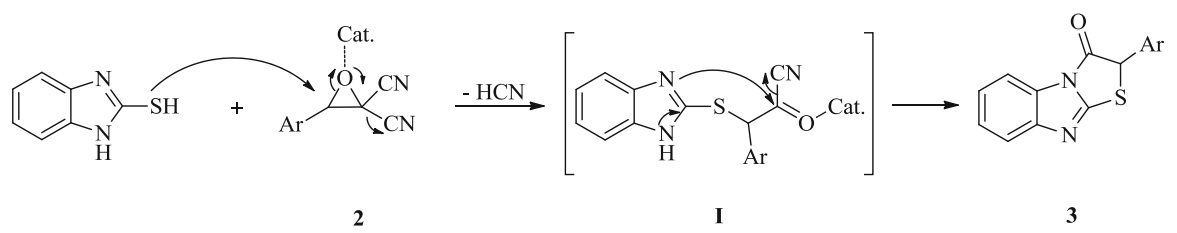

Scheme 2 A reasonable mechanism for formation of product 3

regioselectively on $\mathrm{C}_{\beta}$ and $\mathrm{C}_{\alpha}$ of 2,2-dicyanooxiranes $\mathbf{1}$, which have three electrondeficient centers. These processes are highly regioselective, with the products resulting from initial attack of the nucleophilic sulfur atom of compounds 2,4 or 6 on $\mathrm{C}_{\beta}$ of 2,2-dicyanooxiranes $\mathbf{1}$ followed by ring opening. The intermediate $\mathbf{I}$ was formed by loss of cyano group. The last step is intramolecular nucleophilic addition of nitrogen on carbonyl group of intermediate $\mathbf{I}$, followed by elimination of HCN to form a single product. Coordination of the Lewis acids with the epoxide and carbonyl group oxygen increases the polarity of the $\mathrm{C}-\mathrm{O}$ bond and makes the adjacent carbons more susceptible to nucleophilic attack (Scheme 2).

\section{Conclusions}

Bismuth(III) nitrate pentahydrate and zirconium(IV) chloride are known as effective Lewis acid catalysts for synthesis of heterocyclic compounds which condensed $[1,3]$ thiazole with benzimidazole, triazole or thiazolinone derivatives via nucleophilic reaction of 1,3-dinucleophiles with 2,2-dicyanooxiranes at ambient temperature. These catalysts show an environmentally friendly character and are inexpensive and easily obtained. Moreover, the procedure offers several advantages including high yield, operational simplicity, clean reaction conditions, and short 
reaction time, making it a useful and attractive process for synthesis of these compounds.

\section{Experimental}

General procedures

Melting points were measured on an Electrothermal Engineering Ltd. apparatus and are uncorrected. Infrared (IR) spectra were measured on a Mattson 1000 FT-IR spectrometer. Proton and carbon nuclear magnetic resonance (NMR) spectra were recorded with a Bruker DRX-400 AVANCE spectrometer at 400 and $100 \mathrm{MHz}$, respectively. Mass spectra were recorded on a MS-QP2000A Shimadzu mass spectrometer operating at an ionization potential of $70 \mathrm{eV}$. Elemental analyses were performed using a Heraeus CHN-O-Rapid analyzer. 2,2-Dicyanooxiranes 1a-1d were prepared according to a literature procedure [18].

Procedure for preparation of 2-arylidene-[1,3]thiazolo[3,2-a]benzimidazol-3(2H)ones (3a-3d), 5-substituted thiazolo[3,2-b][1,2,4]triazol-6-ones (5a, 5b), and thiazolinones $(7 \boldsymbol{a}, 7 \boldsymbol{b})$

A mixture of 2,2-dicyanooxiranes 1a-1d (2 mmol), $1 H$-benzo[ $d]$ imidazole-2-thiol 2 or 5-mercapto-3-phenyl-s-triazole 4 or thioureas $(\mathbf{6 a}, \mathbf{6 b})(2 \mathrm{mmol})$, and $\mathrm{ZrCl}_{4}$ $(10 \mathrm{~mol} . \%)$ or $\mathrm{Bi}\left(\mathrm{NO}_{3}\right)_{3} \cdot 5 \mathrm{H}_{2} \mathrm{O}(5 \mathrm{~mol} \%)$ in $\mathrm{CH}_{3} \mathrm{CN}(20 \mathrm{~mL})$ was stirred for the appropriate time (Table 1), the progress of the reaction being monitored by thinlayer chromatography (TLC) using hexane/ethylacetate as eluent. When the reaction was completed as indicated by TLC, the crude product $\mathbf{3 a}-\mathbf{3 d}, \mathbf{5 a}, \mathbf{5 b}, \mathbf{7 a}$, and $\mathbf{7 b}$ was precipitated from the reaction mixture, and the solid was filtered and recrystallized with hexane/ethylacetate to get pure product.

2-Phenyl[1,3]thiazolo[3,2-a]benzimidazol-3(2H)-one (3a) White crystals; m.p. 190-192 ${ }^{\circ} \mathrm{C}$. IR $\left(\mathrm{KBr}, \quad v \max / \mathrm{cm}^{-1}\right): 1,742(\mathrm{C}=\mathrm{O}), 1,603 \quad(\mathrm{C}=\mathrm{N}) .{ }^{1} \mathrm{H} \quad \mathrm{NMR}$ (400 MHz, DMSO-d6): $8.81\left(\mathrm{~d}, 1 \mathrm{H},{ }^{3} \mathrm{~J}_{\mathrm{H}-\mathrm{H}}=8 \mathrm{~Hz}, \mathrm{CH}\right), 7.71 \quad(\mathrm{~d}, 1 \mathrm{H}$, $\left.{ }^{3} \mathrm{~J}_{\mathrm{H}-\mathrm{H}}=8 \mathrm{~Hz}, \mathrm{CH}\right), 7.67-7.39(\mathrm{~m}, 7 \mathrm{H}, \mathrm{Ar}), 6.40(\mathrm{~s}, 1 \mathrm{H}) \cdot{ }^{13} \mathrm{C} \mathrm{NMR}(100 \mathrm{MHz}$, DMSO-d6): $153.84(\mathrm{C}=\mathrm{O}), 144.75(\mathrm{C}=\mathrm{N}), 142.92,133.21,132.69,132.38,129.45$, 129.04, 128.93, 124.63, 122.87, 117.76, $50.10\left(\mathrm{C}_{2}\right)$. MS $(\mathrm{m} / \mathrm{z}): 266\left(\mathrm{M}^{+}(98), 237\right.$ (80), 205 (20), 150 (15), 121 (100), 90 (78), 77 (40). Anal. calcd. for $\mathrm{C}_{15} \mathrm{H}_{10} \mathrm{~N}_{2} \mathrm{OS}$ : C, 67.65; H, 3.78; N, $10.52 \%$. Found: C, 67.42; H, 3.66; N, $10.18 \%$.

2-(4-Chlorophenyl)[1,3]thiazolo[3,2-a]benzimidazol-3(2H)-one (3b) White crystals; m.p. $136-139{ }^{\circ} \mathrm{C}$. IR $\left(\mathrm{KBr}, v \max / \mathrm{cm}^{-1}\right): 1,726(\mathrm{C}=\mathrm{O}), 1,609(\mathrm{C}=\mathrm{N}) .{ }^{1} \mathrm{H}$ NMR (400 MHz, DMSO-d6): $7.87\left(\mathrm{~d}, 1 \mathrm{H},{ }^{3} \mathrm{~J}_{\mathrm{H}-\mathrm{H}}=8 \mathrm{~Hz}, \mathrm{CH}\right), 7.66(\mathrm{~d}, 1 \mathrm{H}$, $\left.{ }^{3} \mathrm{~J}_{\mathrm{H}-\mathrm{H}}=8 \mathrm{~Hz}, \quad \mathrm{CH}\right), \quad 7.62\left(\mathrm{~d}, \quad 2 \mathrm{H}, \quad{ }^{3} \mathrm{~J}_{\mathrm{H}-\mathrm{H}}=8.8 \mathrm{~Hz}, \quad \mathrm{CH}\right), \quad 7.50 \quad(\mathrm{~d}, \quad 2 \mathrm{H}$, $\left.{ }^{3} \mathrm{~J}_{\mathrm{H}-\mathrm{H}}=8.8 \mathrm{~Hz}, \mathrm{CH}\right), 7.42\left(\mathrm{t}, 1 \mathrm{H},{ }^{3} \mathrm{~J}_{\mathrm{H}-\mathrm{H}}=8 \mathrm{~Hz}, \mathrm{CH}\right), 7.35\left(\mathrm{~d}, 1 \mathrm{H},{ }^{3} \mathrm{~J}_{\mathrm{H}-\mathrm{H}}=8 \mathrm{~Hz}\right.$, $\mathrm{CH}), 6.32(\mathrm{~s}, 1 \mathrm{H}) .{ }^{13} \mathrm{C}$ NMR (100 MHz, DMSO-d6): $166.83(\mathrm{C}=\mathrm{O}), 156.88(\mathrm{C}=\mathrm{N})$, 
$149.42,133.61,133.44,131.17,128.72,128.02,125.77,123.74,123.35,118.70$, $67.32\left(\mathrm{C}_{2}\right)$. MS (m/z): $300\left(\mathrm{M}^{+}\right)(100), 27(70), 237(50), 156(80), 124(20), 89$ (73), 63 (40). Anal. calcd. for $\mathrm{C}_{15} \mathrm{H}_{9} \mathrm{ClN}_{2} \mathrm{OS}$ : C, 59.90; H, 3.02; N, $9.31 \%$. Found: C, 59.83; H, 2.88; N, $9.01 \%$.

2-(4-Nitrophenyl)[1,3]thiazolo[3,2-a]benzimidazol-3(2H)-one (3c) White crystals; m.p. $193-196{ }^{\circ} \mathrm{C}$. IR $\left(\mathrm{KBr}, v \max / \mathrm{cm}^{-1}\right)$ : $1,750(\mathrm{C}=\mathrm{O}), 1,609(\mathrm{C}=\mathrm{N}) .{ }^{1} \mathrm{H}$ NMR (400 MHz, DMSO-d6): 8.49 (d, 2H, $\left.{ }^{3} \mathrm{~J}_{\mathrm{H}-\mathrm{H}}=8 \mathrm{~Hz}, \mathrm{CH}\right), 7.72\left(\mathrm{~d}, 1 \mathrm{H},{ }^{3} \mathrm{~J}_{\mathrm{H}-\mathrm{H}}=8 \mathrm{~Hz}\right.$, $\mathrm{CH}), 7.50-7.26(\mathrm{~m}, 5 \mathrm{H}, \mathrm{Ar}), 5.79(\mathrm{~s}, 1 \mathrm{H}) .{ }^{13} \mathrm{C}$ NMR (100 MHz, DMSO-d6): 165.83 $(\mathrm{C}=\mathrm{O}), 153.20(\mathrm{C}=\mathrm{N}), 150.3,145.4,140.01,138.5,129.73,126.56,124.55,124.49$, 119.51, 128.9, $57.61\left(\mathrm{C}_{2}\right) . \mathrm{MS}(\mathrm{m} / \mathrm{z}): 311\left(\mathrm{M}^{+}\right)$(100), $282(20), 265(15), 236(40), 167$ (98), 151 (20), 145 (50), 121 (30), 109 (20), 90 (77), 77 (80), 63 (88). Anal. calcd. for $\mathrm{C}_{15} \mathrm{H}_{9} \mathrm{~N}_{3} \mathrm{O}_{3} \mathrm{~S}: \mathrm{C}, 57.87 ; \mathrm{H}, 2.91 ; \mathrm{N}, 13.50 \%$. Found: C, 57.69; H, 2.85; N, $13.21 \%$.

2-(2,5-Dimethoxyphenyl)[1,3]thiazolo[3,2-a]benzimidazol-3(2H)-one (3d) Yellow crystals; m.p. $140-143{ }^{\circ} \mathrm{C}$. IR $\left(\mathrm{KBr}, v \operatorname{vmax} / \mathrm{cm}^{-1}\right): 1,748(\mathrm{C}=\mathrm{O}), 1,611(\mathrm{C}=\mathrm{N}) .{ }^{1} \mathrm{H}$ NMR (400 MHz, DMSO-d6): $7.81\left(\mathrm{~d}, 1 \mathrm{H},{ }^{3} \mathrm{~J}_{\mathrm{H}-\mathrm{H}}=8 \mathrm{~Hz}, \mathrm{CH}\right), 7.49(\mathrm{~d}, 1 \mathrm{H}$, $\left.{ }^{3} \mathrm{~J}_{\mathrm{H}-\mathrm{H}}=8 \mathrm{~Hz}, \mathrm{CH}\right), 7.31-7.12(\mathrm{~m}, 2 \mathrm{H}, \mathrm{CH}), 7.05(\mathrm{~s}, 1 \mathrm{H}, \mathrm{CH}), 7.02(\mathrm{~d}, 1 \mathrm{H}$, $\left.{ }^{3} \mathrm{~J}_{\mathrm{H}-\mathrm{H}}=8 \mathrm{~Hz}, \mathrm{CH}\right), 6.88\left(\mathrm{~d}, 1 \mathrm{H},{ }^{3} \mathrm{~J}_{\mathrm{H}-\mathrm{H}}=8 \mathrm{~Hz}, \mathrm{CH}\right), 6.01(\mathrm{~s}, 1 \mathrm{H}), 3.65,3.48(\mathrm{~s}, 6 \mathrm{H}$, $\left.2 \mathrm{OCH}_{3}\right) .{ }^{13} \mathrm{C}$ NMR (100 MHz, DMSO-d6): $167.91(\mathrm{C}=\mathrm{O}), 154.70(\mathrm{C}=\mathrm{N}), 152.59$, $150.56,126.40,124.75,124.38,123.38,119.61,117.79,116.30,56.88,56.09$ $\left(2 \mathrm{OCH}_{3}\right) 57.61\left(\mathrm{C}_{2}\right) . \mathrm{MS}(\mathrm{m} / \mathrm{z}): 326\left(\mathrm{M}^{+}\right)(100), 311(85), 267(20), 178(15), 163(50)$, 149 (60), 135 (20), 121 (40), 107 (12), 91 (30), 77 (43), 63 (35). Anal. calcd. for $\mathrm{C}_{17} \mathrm{H}_{14} \mathrm{~N}_{2} \mathrm{O}_{3} \mathrm{~S}$ : C, 62.56; H, 4.32; N, $8.58 \%$. Found: C, 62.44; H, 4.28; N, $8.31 \%$.

5-(4-Chlorophenyl)-2-phenylthiazolo[3,2-b][1,2,4]triazol-6(5H)-one (5a) White crystals; m.p. $152-155^{\circ} \mathrm{C}$. IR $\left(\mathrm{KBr}, \operatorname{vmax} / \mathrm{cm}^{-1}\right)$ : 1,744 $(\mathrm{C}=\mathrm{O}), 1,692,1,611(\mathrm{C}=\mathrm{N}) .{ }^{1} \mathrm{H}$ NMR (400 MHz, DMSO-d6): 8.13-8.11 (m, 2H, Ar), $7.64\left(\mathrm{~d}, 2 \mathrm{H},{ }^{3} \mathrm{~J}_{\mathrm{H}-\mathrm{H}}=8 \mathrm{~Hz}\right.$, $\mathrm{CH}), 7.57-7.53(\mathrm{~m}, 3 \mathrm{H}, \mathrm{Ar}), 7.50\left(\mathrm{~d}, 2 \mathrm{H},{ }^{3} \mathrm{~J}_{\mathrm{H}-\mathrm{H}}=8 \mathrm{~Hz}, \mathrm{CH}\right), 6.41(\mathrm{~s}, 1 \mathrm{H}) .{ }^{13} \mathrm{C}$ NMR (100 MHz, DMSO-d6): $169.73(\mathrm{C}=\mathrm{O}), 165.03,163.96(\mathrm{C}=\mathrm{N}), 134.00$, $132.56,131.35,131.27,129.15,129.04,128.97,126.90,58.17\left(\mathrm{C}_{5}\right) . \mathrm{MS}(\mathrm{m} / \mathrm{z}): 327$ $\left(\mathrm{M}^{+}\right)$(80), 301 (12), 281 (12), 264 (55), 177 (20), 152 (60), 125 (40), 103 (100), 89 (85), 77 (30). Anal. calcd. for $\mathrm{C}_{16} \mathrm{H}_{10} \mathrm{ClN}_{3} \mathrm{OS}$ : C, 58.63; H, 3.07; N, $12.28 \%$. Found: C, 58.44; H, 2.98; N, $11.97 \%$.

5-(2,5-Dimethoxyphenyl)-2-phenylthiazolo[3,2-b][1,2,4]triazol-6(5H)-one (5b) Orange crystals; m.p. $204-206{ }^{\circ} \mathrm{C}$. IR $\left(\mathrm{KBr}, v \max / \mathrm{cm}^{-1}\right): 1,754(\mathrm{C}=\mathrm{O}), 1,606$ $(\mathrm{C}=\mathrm{N}) .{ }^{1} \mathrm{H}$ NMR $\left(400 \mathrm{MHz}\right.$, DMSO-d6): $8.13\left(\mathrm{~d}, 2 \mathrm{H},{ }^{3} \mathrm{~J}_{\mathrm{H}-\mathrm{H}}=8 \mathrm{~Hz}, \mathrm{CH}\right)$, 7.70-7.66 (m, 3H, Ar), 7.20 (s, 1H, CH), 7.05-7.00 (m, 2H, CH), $6.31(\mathrm{~s}, 1 \mathrm{H})$, 3.76, $3.67\left(\mathrm{~s}, 6 \mathrm{H}, 2 \mathrm{OCH}_{3}\right) .{ }^{13} \mathrm{C} \mathrm{NMR}(100 \mathrm{MHz}, \mathrm{DMSO}-\mathrm{d} 6): 169.09(\mathrm{C}=\mathrm{O})$, 164.73, $164.32(\mathrm{C}=\mathrm{N}), 153.08,151.17,131.19,129.10,129.05,126.88,122.58$, 117.28, 115.78, 56.45, $55.84\left(2 \mathrm{OCH}_{3}\right), 55.67\left(\mathrm{C}_{5}\right) . \mathrm{MS}(\mathrm{m} / \mathrm{z}): 353\left(\mathrm{M}^{+}\right)(12), 294$ (7), 178 (30), 163 (95), 147 (20), 135 (23), 121 (15), 103 (100), 92 (18), 77 (28). Anal. calcd. for $\mathrm{C}_{18} \mathrm{H}_{15} \mathrm{~N}_{3} \mathrm{O}_{3} \mathrm{~S}$ : C, 61.18; H, 4.28; N, $11.89 \%$. Found: C, 60.95; H, 4.15; N, $11.60 \%$. 
2-Amino-5-(2,5-dimethoxyphenyl)thiazol-4(5H)-one (7a) Yellow crystals; m.p. 195-196 ${ }^{\circ} \mathrm{C}$. IR $\left(\mathrm{KBr}, \quad v \max / \mathrm{cm}^{-1}\right)$ : 3,316 $\left(\mathrm{NH}_{2}\right),{ }_{1,692}(\mathrm{C}=\mathrm{O}) .{ }^{1} \mathrm{H}$ NMR (400 MHz, DMSO-d6): $8.92\left(\mathrm{~s}, 2 \mathrm{H}, \mathrm{NH}_{2}\right), 7.28\left(\mathrm{~d}, 1 \mathrm{H},{ }^{3} \mathrm{~J}_{\mathrm{H}-\mathrm{H}}=8 \mathrm{~Hz}, \mathrm{CH}\right), 7.15$ $\left(\mathrm{d}, 1 \mathrm{H},{ }^{3} \mathrm{~J}_{\mathrm{H}-\mathrm{H}}=8 \mathrm{~Hz}, \mathrm{CH}\right), 6.98(\mathrm{~s}, 1 \mathrm{H}, \mathrm{CH}), 5.91(\mathrm{~s}, 1 \mathrm{H}, \mathrm{CH}), 3.72,3.65(\mathrm{~s}, 6 \mathrm{H}$, $\left.2 \mathrm{OCH}_{3}\right) .{ }^{13} \mathrm{C}$ NMR (100 MHz, DMSO-d6): $176.25(\mathrm{C}=\mathrm{O}), 169.24(\mathrm{C}=\mathrm{N}), 153.33$, $150.28,127.48,116.15,114.36,113.56,56.25,55.68\left(2 \mathrm{OCH}_{3}\right), 54.94\left(\mathrm{C}_{5}\right) . \mathrm{MS}(\mathrm{m} /$ z): $252\left(\mathrm{M}^{+}\right)$(16), 221 (15), 190 (38), 137 (58), 115 (100), 74 (25). Anal. calcd. for $\mathrm{C}_{11} \mathrm{H}_{12} \mathrm{~N}_{2} \mathrm{O}_{3} \mathrm{~S}: \mathrm{C}, 52.37 ; \mathrm{H}, 4.79 ; \mathrm{N}, 11.10 \%$. Found: C, 52.18; H, 4.39; N, $10.98 \%$.

5-(2,5-Dimethoxyphenyl)-2-(phenylamino)thiazol-4(5H)-one (7b) White crystals; m.p. $218-220{ }^{\circ} \mathrm{C}$. IR $\left(\mathrm{KBr}, v \max / \mathrm{cm}^{-1}\right)$ : 3,303 (NH), 1,702 (C=O). ${ }^{1} \mathrm{H} \mathrm{NMR}$ (400 MHz, DMSO-d6): 9.12 (s, 1H, NH), 7.38 (d, 1H, $\left.{ }^{3} \mathrm{~J}_{\mathrm{H}-\mathrm{H}}=8 \mathrm{~Hz}, \mathrm{CH}\right), 7.27$ (d, $\left.1 \mathrm{H},{ }^{3} \mathrm{~J}_{\mathrm{H}-\mathrm{H}}=8 \mathrm{~Hz}, \mathrm{CH}\right), 7.23-7.02(\mathrm{~m}, 5 \mathrm{H}, \mathrm{Ar}), 6.89(\mathrm{~s}, 1 \mathrm{H}, \mathrm{CH}), 5.78(\mathrm{~s}, 1 \mathrm{H}, \mathrm{CH})$, 3.76, 3.69 (s, 6H, 2OCH $).{ }^{13} \mathrm{C}$ NMR (100 MHz, DMSO-d6): $173.63(\mathrm{C}=\mathrm{O}), 168.23$ $(\mathrm{C}=\mathrm{N}), 153.03,151.10,134.32,128.69,128.45,127.65,124.85,116.87,114.16$, 113.24, 56.45, $55.63\left(2 \mathrm{OCH}_{3}\right), 54.86\left(\mathrm{C}_{5}\right)$. MS ( $\left.\mathrm{m} / \mathrm{z}\right): 328\left(\mathrm{M}^{+}\right)(10), 297(26), 266$ (16), 251 (58), 191 (12), 189 (26), 137 (34), 77 (100). Anal. calcd. for $\mathrm{C}_{17} \mathrm{H}_{16} \mathrm{~N}_{2} \mathrm{O}_{3} \mathrm{~S}$ : C, 62.18; H, 4.91; N, $8.53 \%$. Found: C, 61.97; H, 4.48; N, $8.12 \%$.

Acknowledgments The authors express appreciation to the Shahid Bahonar University of Kerman Faculty Research Committee for its support of this investigation.

Open Access This article is distributed under the terms of the Creative Commons Attribution License which permits any use, distribution, and reproduction in any medium, provided the original author(s) and the source are credited.

\section{References}

1. A. Majcen-Le Marechal, A. Robert, I. Leban, Tetrahedron 46, 453 (1990)

2. A. Souizi, A.C.R. Robert, Acad. Sci., Paris Ser. 295, 571 (1982)

3. M. Guillemet, A. Robert, M. Baudy-Floch, Tetrahedron Lett. 36, 547 (1995)

4. A. Majcen-Le Marechal, A. Robert, I. Leban, J. Chem. Soc., Perkin Trans. 1, 351 (1993)

5. A. Majcen-Le Marechal, P. Le Grel, A. Robert, J. Biskup, V. Ferk, R. Toplak, Arkivoc v, 119 (2001)

6. J.A. Deyrup, C.L. Moyer, J. Org. Chem. 34, 175 (1969)

7. G.H. Posner, D.Z. Rogers, J. Am. Chem. Soc. 99, 8208 (1977)

8. G. Sekar, V.K. Singh, J. Org. Chem. 64, 287 (1999)

9. S. Chandrasekhar, T. Ramchander, S.J. Prakash, Synthesis 1817 (2000)

10. M. Chini, P. Crotti, F. Macchia, Tetrahedron Lett. 31, 4661 (1990)

11. H. Kotsuki, K. Hayashida, T. Shimanouchi, H. Nishizawa, J. Org. Chem. 61, 948 (1996)

12. M.M. Mojtahedi, M.R. Saidi, M. Bolourtchian, J. Chem. Res. 128 (1999)

13. M.A.P. Martins, M.V.M. Teixeira, V. Cunico, E. Scapin, R. Mayer, M.P. Pereira, N. Zanatta, H.G.

Bonacorso, C. Peppe, Y.F. Yuan, Tetrahedron Lett. 45, 8991 (2004)

14. H. Suzuki, T. Ikegami, Y. Matano, Synthesis 249 (1997)

15. K. Asit, A.K. Chakraborti, A. Kondaskar, Tetrahedron Lett. 44, 8315 (2003)

16. P.J. Moles, Adhesion Sci. Technol. 6, 61 (1992)

17. M. Seifi, H. Sheibani, Arkivoc iv, 191 (2013)

18. J. Volmajer, R. Toplak, S. Bittner, A.M.L. Marechal, Arkivoc xiv, 49 (2003)

19. S. Jaguelin, A. Robert, P. Gayral, Eur. J. Med. Chem. 26, 51 (1991) 